\title{
Ginsenoside Rb1 Treatment Attenuates Pulmonary Inflammatory Cytokine Release and Tissue Injury following Intestinal Ischemia Reperfusion Injury in Mice
}

\author{
Ying Jiang, ${ }^{1}$ Zhen Zhou, ${ }^{2}$ Qing-tao Meng, ${ }^{1}$ Qian Sun, ${ }^{1}$ Wating Su, \\ Shaoqing Lei, ${ }^{1}$ Zhengyuan Xia, ${ }^{3,4}$ and Zhong-yuan Xia ${ }^{1}$ \\ ${ }^{1}$ Department of Anesthesiology, Renmin Hospital of Wuhan University, Wuhan 430060, China \\ ${ }^{2}$ Department of Cardiovascular Surgery, Renmin Hospital of Wuhan University, Wuhan 430060, China \\ ${ }^{3}$ Department of Anesthesiology, The University of Hong Kong, Pok Fu Lam, Hong Kong \\ ${ }^{4}$ Department of Anesthesiology, Affiliated Hospital of Guangdong Medical College, Zhanjiang, Guangdong 524001, China \\ Correspondence should be addressed to Zhong-yuan Xia; xiazhongyuan2005@aliyun.com
}

Received 18 September 2014; Revised 22 December 2014; Accepted 27 December 2014

Academic Editor: Yanfang Chen

Copyright ( 2015 Ying Jiang et al. This is an open access article distributed under the Creative Commons Attribution License, which permits unrestricted use, distribution, and reproduction in any medium, provided the original work is properly cited.

\begin{abstract}
Objective. Intestinal ischemia reperfusion (II/R) injury plays a critical role in remote organ dysfunction, such as lung injury, which is associated with nuclear factor erythroid 2-related factor 2 (Nrf2)/heme oxygenase-1 (HO-1) signaling pathway. In the present study, we tested whether ginsenoside Rb1 attenuated II/R induced lung injury by Nrf2/HO-1 pathway. Methods. II/R injury was induced in male C57BL/6J mice by 45 min of superior mesenteric artery (SMA) occlusion followed by 2 hours of reperfusion. Ginsenoside $\mathrm{Rb} 1$ was administrated prior to reperfusion with or without ATRA (all-transretinoic acid, the inhibitor of Nrf2/ARE signaling pathway) administration before II/R. Results. II/R induced lung histological injury, which is accompanied with increased levels of malondialdehyde (MDA), interleukin- (IL-) 6, and tumor necrosis factor- (TNF-) $\alpha$ but decreased levels of superoxide dismutase (SOD) and IL-10 in the lung tissues. Ginsenoside Rb1 reduced lung histological injury and the levels of TNF- $\alpha$ and MDA, as well as wet/dry weight ratio. Interestingly, the increased Nrf2 and HO-1 expression induced by II/R in the lung tissues was promoted by ginsenoside Rbl treatment. All these changes could be inhibited or prevented by ATRA. Conclusion. Ginsenoside Rbl is capable of ameliorating II/R induced lung injuries by activating Nrf2/HO-1 pathway.
\end{abstract}

\section{Introduction}

Intestinal ischemia reperfusion (II/R) injury is a lifethreatening clinical surgical emergency, which is associated with the exacerbation of intestinal injury and a systemic inflammatory response leading to progressive distal organ impairment, finally resulting in cardiocirculatory, respiratory, hepatic, and renal failure. Acute respiratory distress syndrome (ARDS) induced by lung injury is one of the most serious complications. These clinical problems were involved in diverse causes such as intestinal barrier damage, bacteria translocation and oxidative stress, and activation of multiple inflammatory mediators $[1,2]$. However, there still remain many doubts in the pathophysiology and therapeutics of II/R induced remote organ injury, especially lung injury.
Ginsenoside Rb1, a major active constituent of ginseng (Panax ginseng), has antioxidative effects and has been demonstrated to protect multiple organs from ischemia reperfusion injury [3-9]. However, it has not been fully elucidated whether it can also attenuate II/R induced acute lung injury. Nuclear factor erythroid 2-related factor 2 (Nrf2)/antioxidant response element (ARE) signaling pathway has been found as the most important endogenous antioxidative stress mechanism. It has been reported that Nrf2/ARE signaling pathway performs a fundamental role in protecting the body against the xenobiotics and oxidative injury in the pathophysiology of digestive system, circulation system, nervous system, and immune system diseases [1013]. Nrf2 is a nuclear transcription factor that controls the expression and coordinates induction of a battery of defensive 
genes encoding detoxifying enzymes and antioxidant proteins [14]. In response to stimulation of oxidative stress, Nrf2 translocates from the cytoplasm into the nucleus and then binds to a cis-acting enhancer sequence designated as ARE and regulates ARE mediated antioxidant enzyme gene such as heme oxygenase-1 (HO-1) expression and induction $[15,16]$. HO-1 belongs to a member of the heat shock protein family and plays a significant protective role against inflammatory processes and oxidative tissue injury [17].

In this study, we established a superior mesenteric artery (SMA) occlusion/reperfusion mice model to induce lung injury. We used ATRA (all-transretinoic acid) as inhibitor of Nrf2/ARE signaling pathway, which interfered in the recruitment of Nrf2 to the ARE, thus disrupting the activation of ARE-driven genes [18]. With the treatment of ginsenoside $\mathrm{Rb}$, we aim to investigate whether ginsenoside Rbl attenuates acute lung injury (ALI) induced by II/R in mice via Nrf2/ARE pathway.

\section{Material and Methods}

2.1. Mice. The current study was approved by the Animal Care Committee of Wuhan University, China, and was performed in accordance with National Institutes of Health guidelines for the use of experimental animals. Male C57BL/6 mice (9-12 weeks old; 17-22 g) were purchased from HUNAN SLAC JD Laboratory Animal Co. Ltd., China. They were housed under standard laboratory conditions at 22$24^{\circ} \mathrm{C}$, relative humidity of $50 \pm 15 \%$, and kept on a $12 \mathrm{~h}$ day/night rhythm with free access to water and food. All experimental protocols conducted in the mice were carried out in accordance with the Guide for the Care and Use of Laboratory Animals by the National Institutes of Health (NIH Publication number 80-23).

2.2. Surgical Preparation. Animals were anesthetized intraperitoneally with pentobarbital sodium $(50 \mathrm{mg} / \mathrm{kg}$ body weight). A midline laparotomy was performed; then the superior mesenteric artery (SMA) was isolated. The II/R injury was established by occluding SMA with a microvascular clip for 45 minutes followed by 2 hours of reperfusion as previously described [19]. Ischemia was recognized by the existence of pulseless or pale color of the small intestine. The return of pulses and the reestablishment of the pink color were assumed to indicate valid reperfusion of the intestine. The Sham group underwent the same surgical process, apart from occlusion of SMA. After $2 \mathrm{~h}$ reperfusion, the mice were killed. A median sternotomy was performed; the lung and intestine samples were obtained for further analysis.

2.3. Experimental Protocol. The mice were randomly allocated into eight groups ( $n=8$ in each group) (Figure 1 ): (1) Sham surgical preparation including isolation of the SMA without occlusion was performed (Sham); (2) mice were subjected to II/R without treatment (II/R); (3) mice were subjected to II/R with treatment of normal saline 10 minutes before reperfusion (II/R + NS); (4), (5) mice were treated
(1) Sham

(2) $I / / R$

(3) $I I / R+N S$

(4) $\mathrm{II} / \mathrm{R}+\mathrm{Rb} 1-30$

(5) $\mathrm{II} / \mathrm{R}+\mathrm{Rb} 1-60$

(6) ATRA + sham

(7) ATRA + II/R

(8) ATRA + II/R + Rb-60

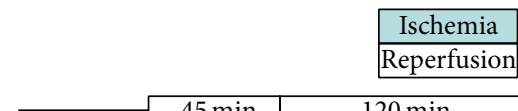

\begin{tabular}{|c|c|c|}
\hline & $45 \mathrm{~min}$ & $120 \mathrm{~min}$ \\
\hline & $45 \mathrm{~min}$ & $120 \mathrm{~min}$ \\
\hline & & $\sqrt{ }$ Normal saline \\
\hline & $45 \mathrm{~min}$ & $120 \mathrm{~min}$ \\
\hline & & $\sqrt{-} \mathrm{Rb} 1(30 \mathrm{mg} / \mathrm{kg})$ \\
\hline & $45 \mathrm{~min}$ & $120 \mathrm{~min}$ \\
\hline & & $\sqrt{ }-\mathrm{Rb} 1(60 \mathrm{mg} / \mathrm{kg})$ \\
\hline & $45 \mathrm{~min}$ & $120 \mathrm{~min}$ \\
\hline ATRA & p. 2 weeks & \\
\hline$\downarrow$ & $45 \min$ & $120 \mathrm{~min}$ \\
\hline
\end{tabular}

ATRA i.p. 2 weeks

v \begin{tabular}{r|r|}
\hline $45 \mathrm{~min}$ & $120 \mathrm{~min}$ \\
\hline
\end{tabular}

ATRA i.p. 2 weeks $\mathrm{Rb} 1(60 \mathrm{mg} / \mathrm{kg})$

\begin{tabular}{r|r}
$\sqrt{ }$ \\
$45 \mathrm{~min}$ & $120 \mathrm{~min}$ \\
\hline
\end{tabular}

FIGURE 1: Experimental protocols. Mice were subjected to $45 \mathrm{~min}$ of SMA occlusion followed by $2 \mathrm{~h}$ of reperfusion. II/R: intestinal ischemia/reperfusion, NS: normal saline, Rb1: ginsenoside Rb1, and ATRA: all-transretinoic acid.

with $30 \mathrm{mg} / \mathrm{kg}(\mathrm{II} / \mathrm{R}+\mathrm{Rb} 1-30)$ or $60 \mathrm{mg} / \mathrm{kg}$ (II/R + Rb160) ginsenoside Rb1, in which surgery was performed as in the II/R group with administration of the ginsenoside Rb1 intraperitoneally 10 minutes before reperfusion; (6) mice were subjected to Sham surgery and treated with ATRA (ATRA + Sham), which is the inhibitor of Nrf2/ARE signaling pathway; (7) mice were subjected to II/R and treated with ATRA (ATRA + II/R); (8) mice were subjected to II/R and treated with ATRA and $60 \mathrm{mg} / \mathrm{kg}$ ginsenoside $\mathrm{Rb} 1$ as group 5 (ATRA + II/R + Rb1-60). During the last two weeks before the operation, the mice in the group 6, 7, 8 received ATRA i.p. daily at $10 \mathrm{mg} / \mathrm{kg}$ and fed on a vitamin A-deficient diet, and the mice in the other groups received the equivalent volume of corn oil and fed on a control normal diet [18].

2.4. Lung Histology. The left lung was removed and fixed in $10 \%$ formalin. Following embedding in paraffin, the sections of $4 \mu \mathrm{m}$ were stained with hematoxylin and eosin for light microscopy. Semiquantitative analysis of lung histopathology was performed by scoring the tissues based on lung edema, infiltration of inflammatory cells, alveolar hemorrhage, hyaline membrane, and atelectasis: no lesion, 0 ; injured area $\leqslant$ $25 \%, 1$; injured area $26-50 \%, 2$; injured area $51-70 \%, 3$; injured area $71-90 \%, 4$; injured area $>90 \%, 5$. A total of three fields were randomly selected for each slide and the average was used as the histopathology score [20].

2.5. Histopathological Assessment of Intestines. After reperfusion, $1 \mathrm{~cm}$ of small intestine without adipose tissue was taken from the same place at the distal end of ileum and fixed in $4 \%$ formaldehyde. After embedding in paraffin, $4 \mu \mathrm{m}$ sections were stained with hematoxylin and eosin before assessment by light microscopy (original magnification $\times 200$, Olympus BX50; Olympus Optical, Tokyo, Japan). 


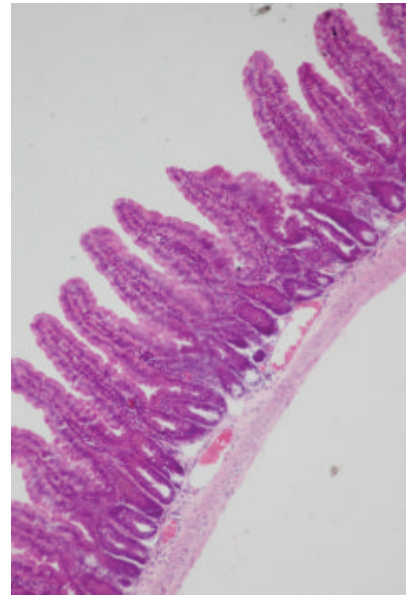

(a)

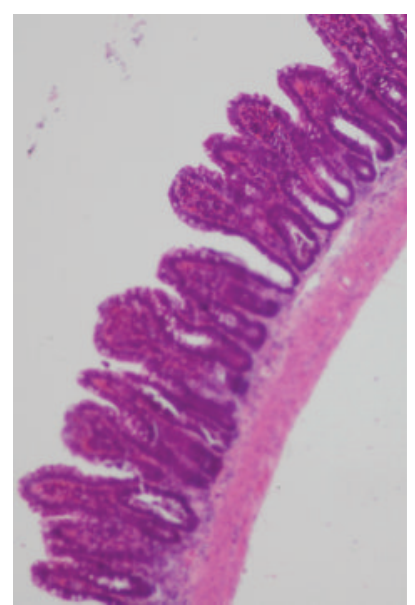

(e)

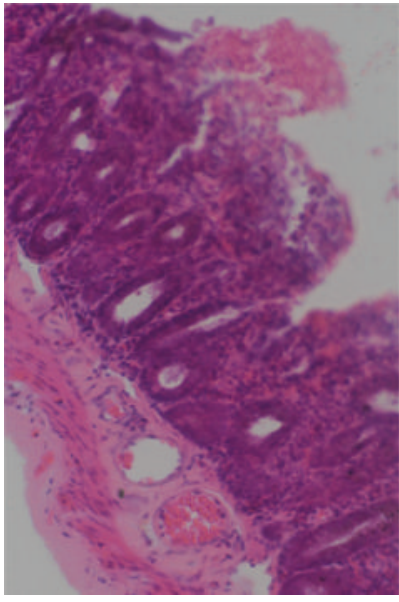

(b)

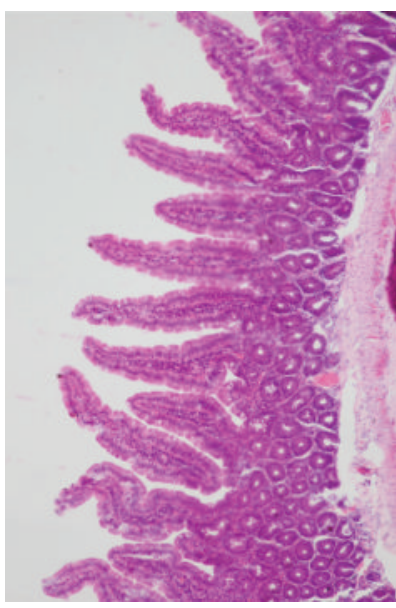

(f)

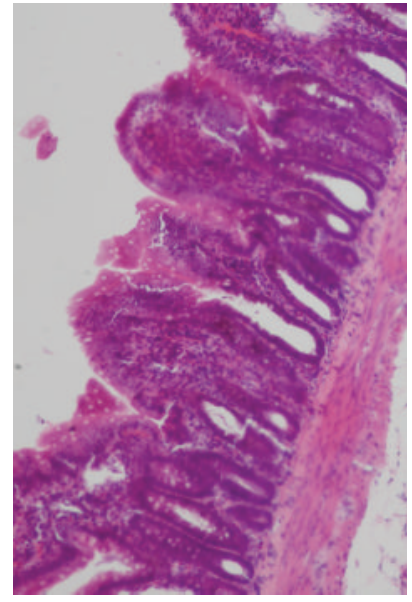

(c)

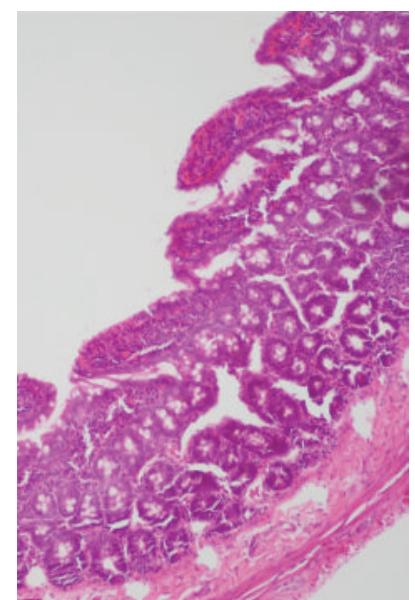

(g)

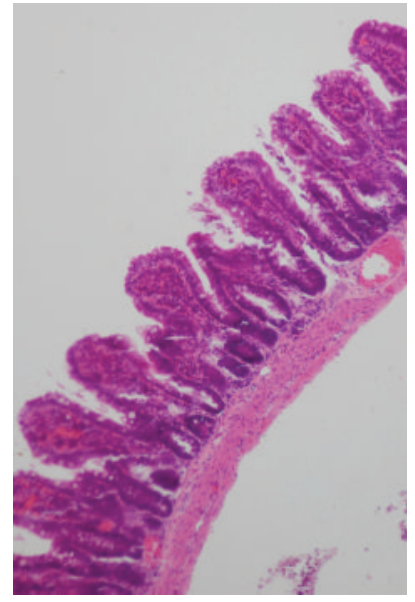

(d)

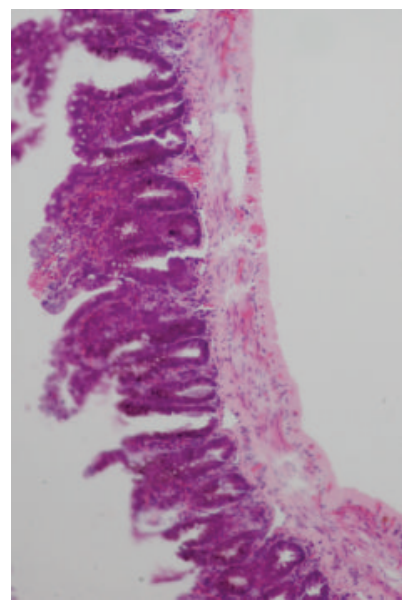

(h)

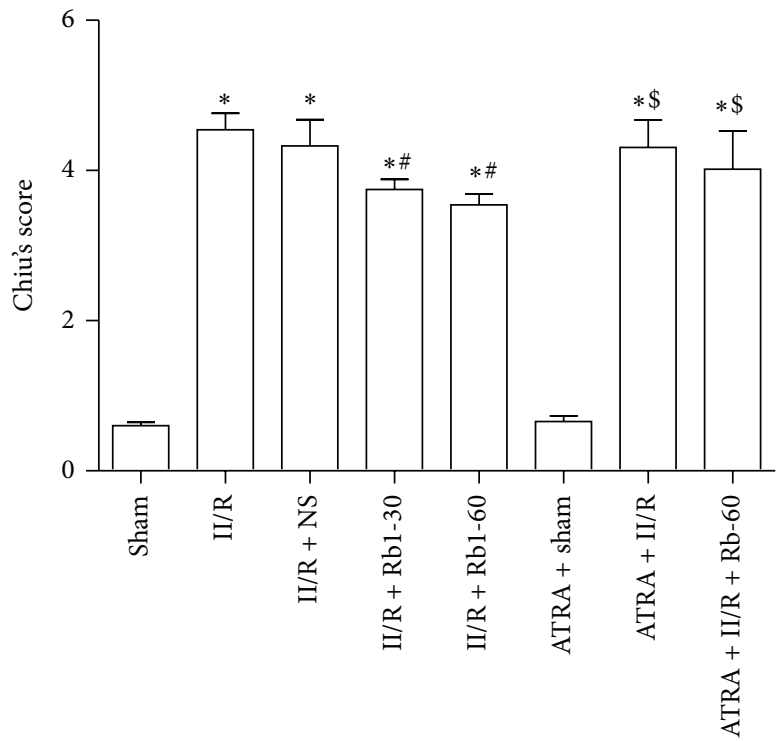

(i)

FIgURE 2: Intestinal histologic evaluation in the groups. ((a)-(h)) Histopathologic changes of the small-intestinal mucosa were observed under light microscopy (hematoxylin and eosin, $\times 200$ ). (a) Sham group, (b) II/R group, (c) II/R + NS group, (d) II/R + Rb1-30 group, (e) II/R + Rbl-60 group, (f) ATRA + Sham group, (g) ATRA + II/R group, and (h) ATRA + II/R + Rbl-60 group. Data are mean \pm SD, $n=10$; ${ }^{*} P<0.05$ versus Sham group, ${ }^{\#} P<0.05$ versus II/R group, and ${ }^{\$} P<0.05$ versus $\mathrm{II} / \mathrm{R}+\mathrm{Rb1}-60$ group. 


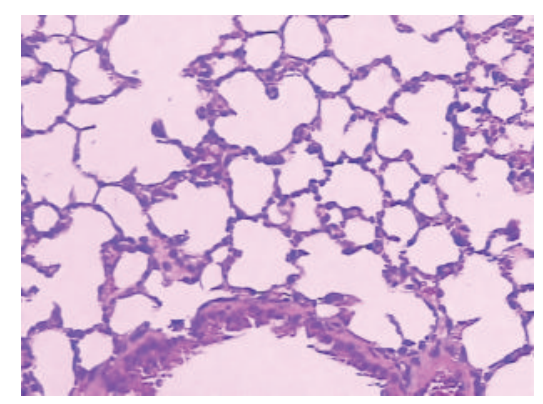

(a)

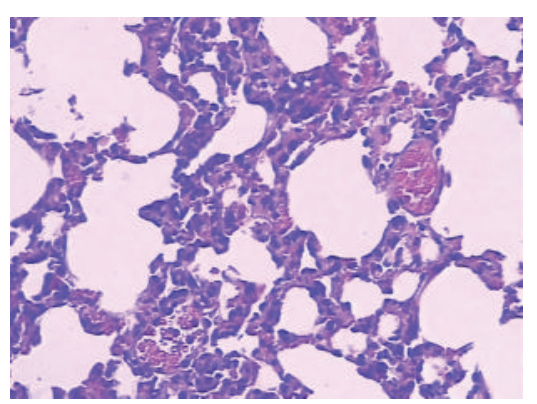

(d)

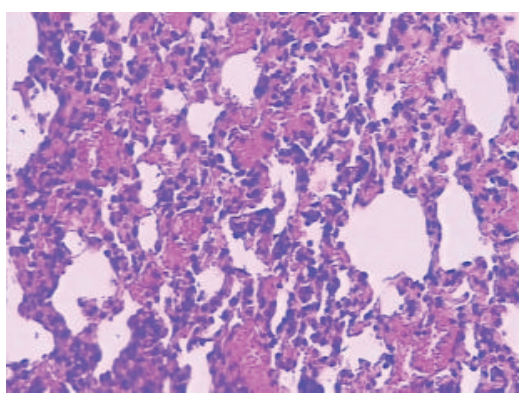

(b)

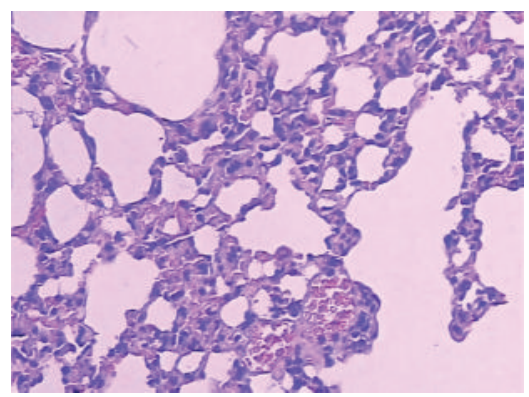

(e)

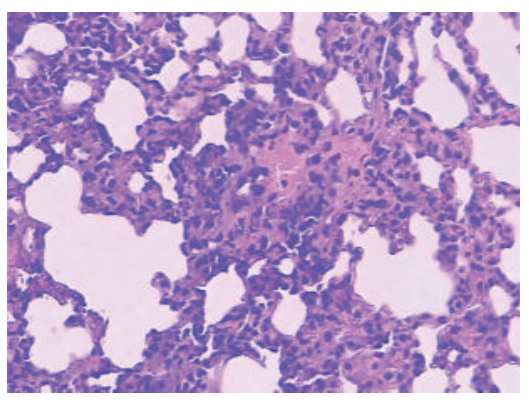

(c)

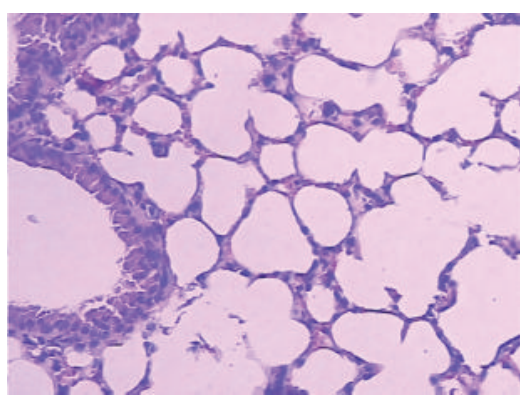

(f)

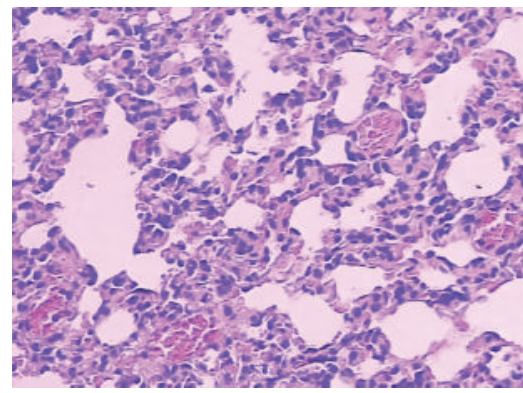

(g)

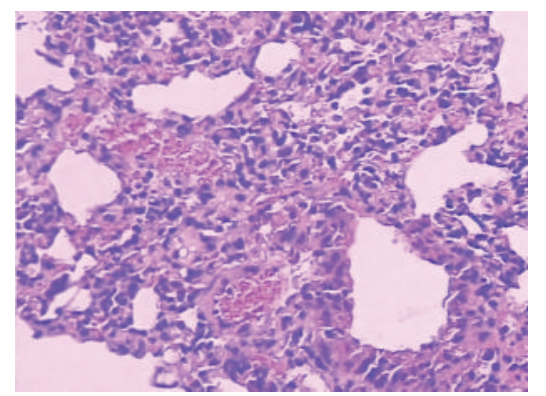

(h)

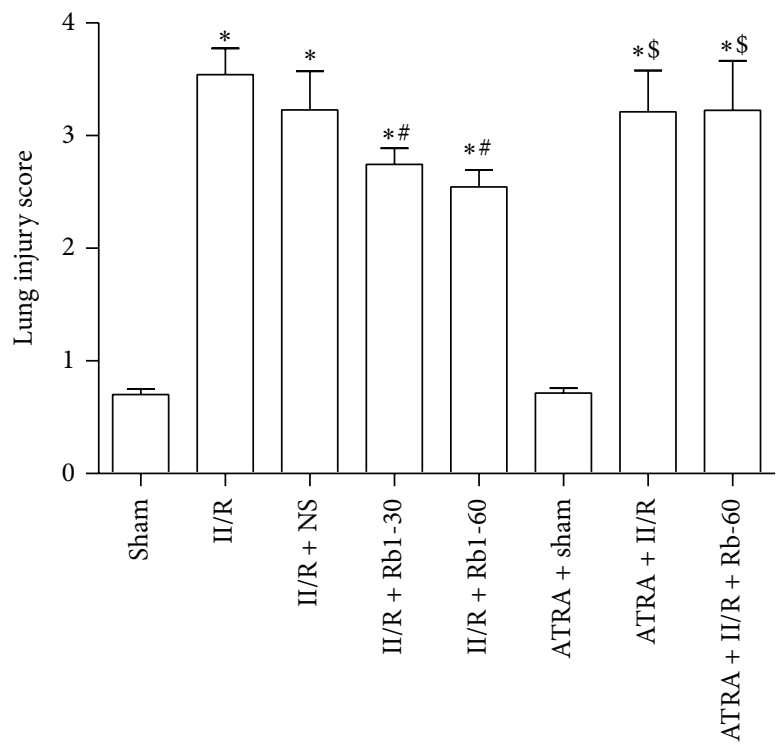

(i)

FIGURE 3: Histopathologic changes in mice lung under light microscopy (hematoxylin and eosin, $\times 200$ ). (a) Sham group, (b) II/R group, (c) II/R + NS group, (d) II/R + Rbl-30 group, (e) II/R + Rbl-60 group, (f) ATRA + Sham group, (g) ATRA + II/R group, and (h) ATRA + II/R + Rbl-60 group. Data are mean $\pm \mathrm{SD}, n=10 ;{ }^{*} P<0.05$ versus Sham group, ${ }^{*} P<0.05$ versus II/R group, and ${ }^{\$} P<0.05$ versus II/R $+\mathrm{Rb} 1-60$ group. 


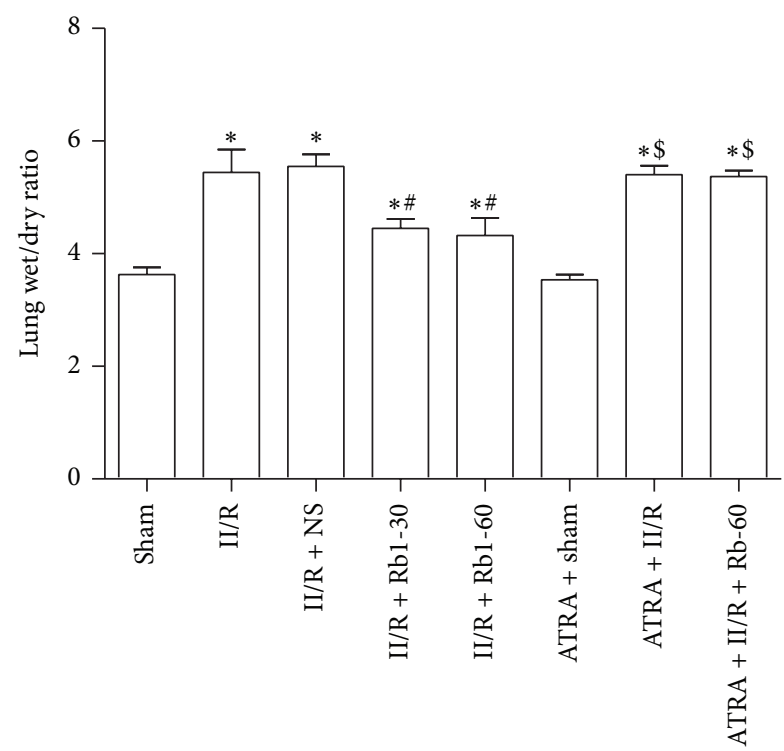

FIGURE 4: The effects of ginsenoside Rb1 on the lung wet/dry weight ratio. Data are mean $\pm \mathrm{SD}, n=5 ;{ }^{*} P<0.05$ versus Sham group, ${ }^{\#} P<0.05$ versus II/R group, and ${ }^{\$} P<0.05$ versus II/R + Rb1-60 group.

Using the improved Chiu score method [21] to evaluate intestinal mucosal damage, higher scores are interpreted to indicate more severe damage. Criteria of Chiu grading system consist of 5 subdivisions according to the changes of villus and gland of intestinal mucosa: grade 0 , normal mucosa; grade 1, development of subepithelial Gruenhagen's space at the tip of villus; grade 2 , extension of the space with moderate epithelial lifting; grade 3, massive epithelial lifting with a few denuded villi; grade 4, denuded villi with exposed capillaries; and grade 5, disintegration of the lamina propria, ulceration, and hemorrhage.

2.6. Assessment of Pulmonary Edema. The left lung was harvested. After the lung wet weight was measured, the lungs were placed in a calorstat at $60^{\circ} \mathrm{C}$ for $48 \mathrm{~h}$, and then the specimen was reweighed. The pulmonary edema was estimated by lung wet/dry weight ratio [22].

2.7. Immunohistochemical Assessment. Paraffin-embedded lung sections were stained using the streptavidin-biotin complex immunohistochemistry technique for HO-1 and Nrf2 detection. Brown staining in the cytoplasm and/or nucleus was considered an indicator of positive expression. With the Image-Pro Plus version 6.0, results were evaluated semiquantitatively according to optical density values of positive expression.

2.8. Western Blot Analysis. The right lungs were removed and nuclear fractions were prepared using a Nuclear and Cytoplasmic Protein Extraction Kit (Beyotime Institute of Biotechnology, China) according to the manufacturer's protocol. Western blot analysis was carried out as described [23]. Primary antibodies (working concentration) used were rabbit polyclonal antibodies against mice Nrf2 (1:2000, H-300,
Santa Cruz Biotechnology, CA), HO-1 (1:1000, H-105, Santa Cruz Biotechnology, CA), and Lamin B1 (1:2000, H-90, Santa Cruz Biotechnology, CA). The HRP-conjugated secondary antibody was goat anti-rabbit IgG (Beyotime Institute of Biotechnology, China) used at 1:2000. Lamin B was used as an internal control. The ECL Western blotting detection reagents (Beyotime Institute of Biotechnology, China) were used for visualization of the protein bands. The intensities of the bands were analyzed with quantity one software (Bio-Rad, Hercules, CA).

2.9. Determination of Tissue Tumor Necrosis Factor Alpha (TNF- $\alpha$ ), Interlukin-6 (IL-10), and Interlukin-6 (IL-6). Tissue levels of TNF- $\alpha$, IL-10, and IL- 6 were determined using commercially available ELISA kits (R\&D, Minneapolis, MN) according to manufacturer's instructions. The results were expressed as $\mathrm{pg} / \mathrm{mL}$.

2.10. Determination of Tissue MDA Level and SOD Activity. The right lung tissues were homogenized on ice in normal saline. The homogenates were centrifuged at $4000 \mathrm{~g} \cdot \mathrm{min}^{-1}$ at $4^{\circ} \mathrm{C}$ for $10 \mathrm{~min}$. The $\mathrm{MDA}$ level in the supernatants was determined by the measurement of thiobarbituric acidreactive substances levels (assay kits were supplied by Nanjing Jiancheng Corp., China) as previously described [24]. The results were calculated as nmol.100 $\mathrm{mg}^{-1}$ protein. The SOD activity in the supernatants was evaluated by inhibition of nitroblue tetrazolium (NBT) reduction by $\mathrm{O}^{2-}$ generated by the xanthine/xanthine oxidase system (assay kits were supplied by Nanjing Jiancheng Corp., China) in accordance with the previous method [24]. The results were expressed by $\mathrm{U} \cdot 100 \mathrm{mg}^{-1}$ protein.

2.11. Statistical Analysis. Data are presented as mean \pm SD. Statistical comparison among multiple groups was performed by one-way analysis of variance (ANOVA) followed by Newman-Keuls multiple comparison test using the GraphPad Prism 5.0 software (GraphPad Software Inc., San Diego, CA, USA). A value of $P<0.05$ was considered statistically significant.

\section{Results}

3.1. Histopathological Assessment of Intestines. The II/R group showed edema in the villi, inflammatory cells infiltration, and damaged areas interspersed with hemorrhage. In addition, the gap between epithelial cells significantly increased and capillaries and lymph vessels were markedly dilated. Normal villi were seen in the intestine of the Sham group and ATRA + Sham group under the light microscope. Ginsenoside Rb1 at the dose of $30 \mathrm{mg} / \mathrm{kg}$ and $60 \mathrm{mg} / \mathrm{kg}$ both significantly attenuated the histological intestine injury (Figure 2). However, there is little amelioration of the intestine injury induced by II/R in the ATRA + II/R + Rbl-60 group.

3.2. Pathologic Alternations of Lung Tissue. The lungs from II/R group showed damaged areas interspersed with hemorrhage, inflammatory cell infiltration, and pulmonary edema, 


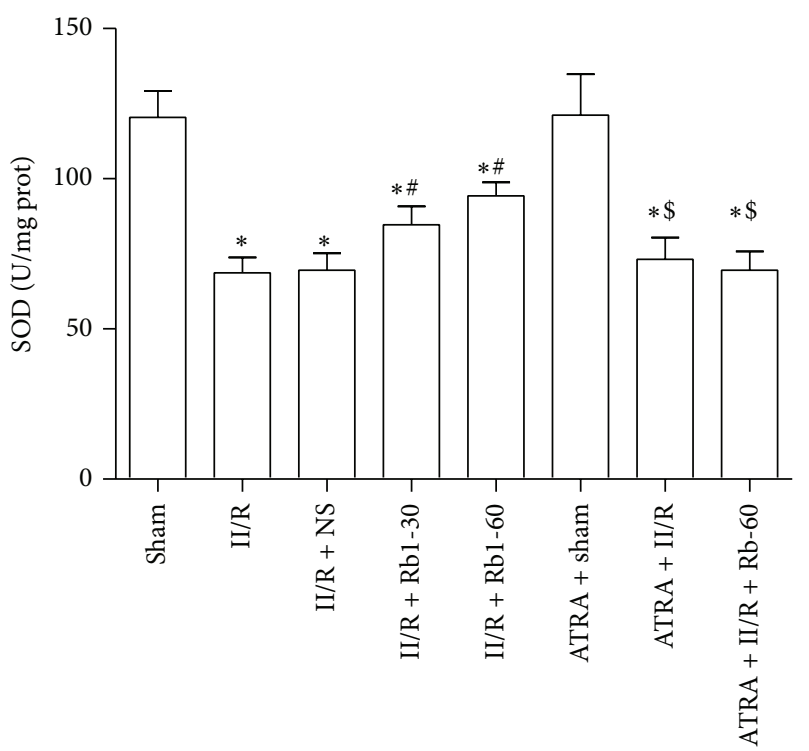

(a)

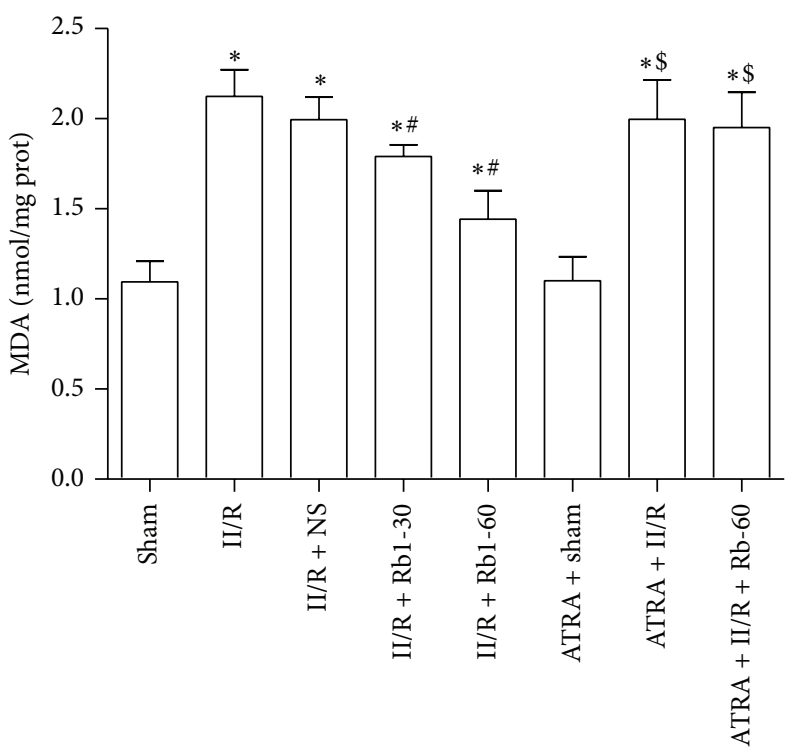

(b)

Figure 5: The changes of lung tissue SOD activity and MDA levels. Data are mean $\pm \mathrm{SD}, n=10 ;{ }^{*} P<0.05$ versus Sham group, ${ }^{\#} P<0.05$ versus II/R group, and ${ }^{\$} P<0.05$ versus II/R + Rb1-60 group.

while little damage was seen in the lungs of the sham group and ATRA + Sham group under the light microscope. Ginsenoside Rbl at the both doses of $30 \mathrm{mg} / \mathrm{kg}$ and $60 \mathrm{mg} / \mathrm{kg}$ significantly attenuated the histological lung injury (Figure 3). However, there is little amelioration of the lung injury induced by II/R in the ATRA + II/R + Rb1-60 group. This indicates that ATRA attenuated the protective action of ginsenoside $\mathrm{Rb}$ against II/R induced lung damage in the mice.

3.3. Changes of Lung Wet/Dry Weight Ratio. We next assessed the lung wet/dry weight ratio as indicators of lung permeability damage. As shown in Figure 4, the lung wet/dry weight ratio was significantly higher in the II/R group than the Sham group $(P<0.05)$. Compared with the II/R group, the lung wet/dry ratio was decreased significantly after the treatment of ginsenoside $\mathrm{Rb} 1(P<0.05, \mathrm{II} / \mathrm{R}+\mathrm{Rb} 1-30$ or II/R + Rb1-60 versus II/R). This decrease was reversed by administration of ATRA $(P>0.05$, ATRA + II/R + Rb1-60 versus II/R). There was no significant difference between the Sham and ATRA + Sham group or II/R and II/R + NS group $(P>0.05)$.

3.4. Changes of the Level of MDA and the Activity of SOD. Oxidative stress has been proposed as an important mechanism of the development of II/R induced organ damage. Reperfusion or reoxygenation will activate recovery of aerobic metabolism and results in an overload of reactive oxygen species (ROS). Robust ROS generation generates excessive hydroxyl radicals, which are very unstable and have a high potential to damage cellular structures, enzymes, or channel proteins on the cellular membrane. We examined the effects of ginsenoside $\mathrm{Rb}$ l on lung tissue lipidic peroxidation product (MDA) levels and the antioxidative SOD activity. As shown in
Figure 5, treatment with $30 \mathrm{mg} / \mathrm{kg}$ and $60 \mathrm{mg} / \mathrm{kg}$ ginsenoside Rb1 significantly reduced MDA levels and increased SOD activity, and this effect was inhibited by administration of ATRA.

3.5. Changes of Tissue TNF- $\alpha$, IL-10, and IL-6 Levels. In a number of clinical studies, microinflammation has been found to be associated with processes that may be related to II/R caused injury. As shown in Figure 6, the level of tissues TNF- $\alpha$ and IL- 6 in the II/R group was significantly higher than that in the Sham group. However, the level of tissue IL-10 was significantly reduced in the II/R group compared to that in the Sham group. Treatment with $30 \mathrm{mg} / \mathrm{kg}$ and $60 \mathrm{mg} / \mathrm{kg}$ ginsenoside Rb1 significantly reduced TNF- $\alpha$ and IL- 6 levels and increased IL-10 levels. After treatment with ATRA, this effect was inhibited.

3.6. Effects of Ginsenoside Rb1 on HO-1 and Nrf2 Expression in Lung Tissue by Immunohistochemical Detection. The lung tissue was obtained to measure the expression of Nrf2 and HO-1 by immunohistochemical assay. In the II/R group, both cytoplasm and nuclei of the lung tissue showed Nrf2 expression, but the expression of $\mathrm{HO}-1$ was showed in the cytoplasm. Compared with the Sham group, the expression of Nrf2 and HO-1 in the II/R group increased significantly. After treatment with ginsenoside Rb1 at dose 30 or $60 \mathrm{mg} / \mathrm{kg}$, the expression of Nrf2 and $\mathrm{HO}-1$ was much higher than that in the II/R group. In the ATRA + II/R and ATRA + II/R + Rb1-60 groups, Nrf2 was also expressed obviously in the cytoplasm and nuclei, though mild expression of $\mathrm{HO}-1$ could be seen in the cytoplasm of lung tissue in these groups (Figures 7 and 8). 


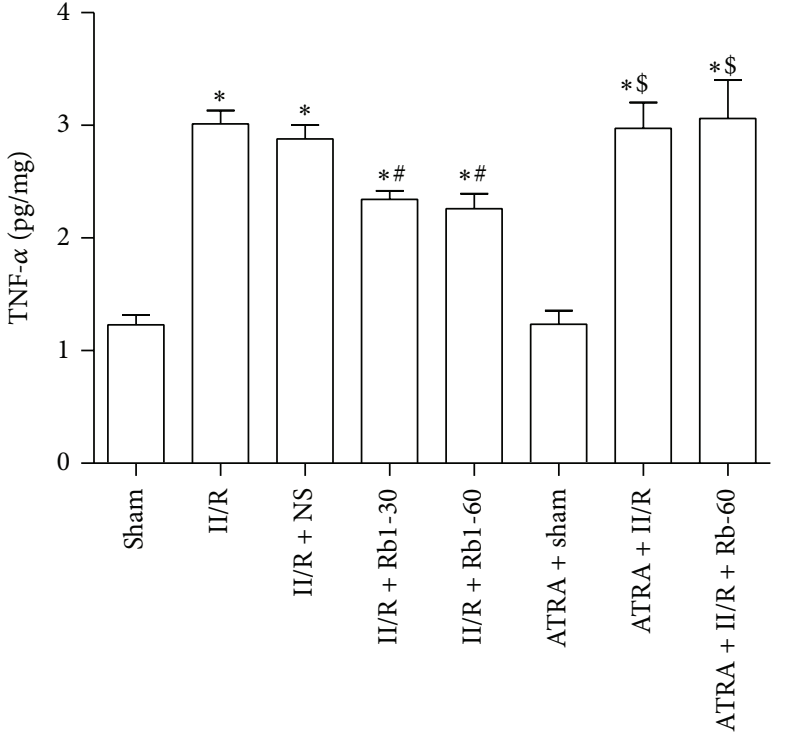

(a)

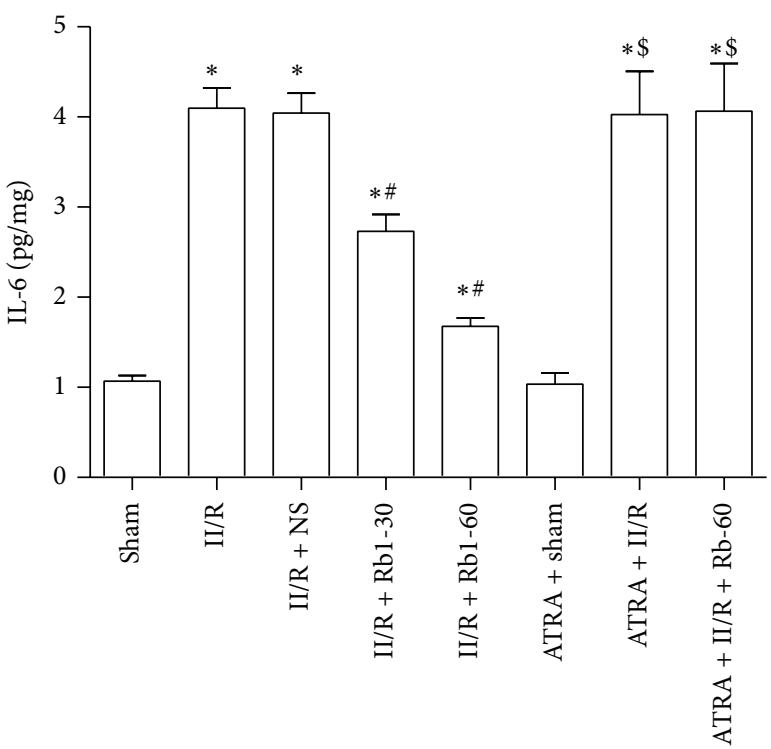

(b)

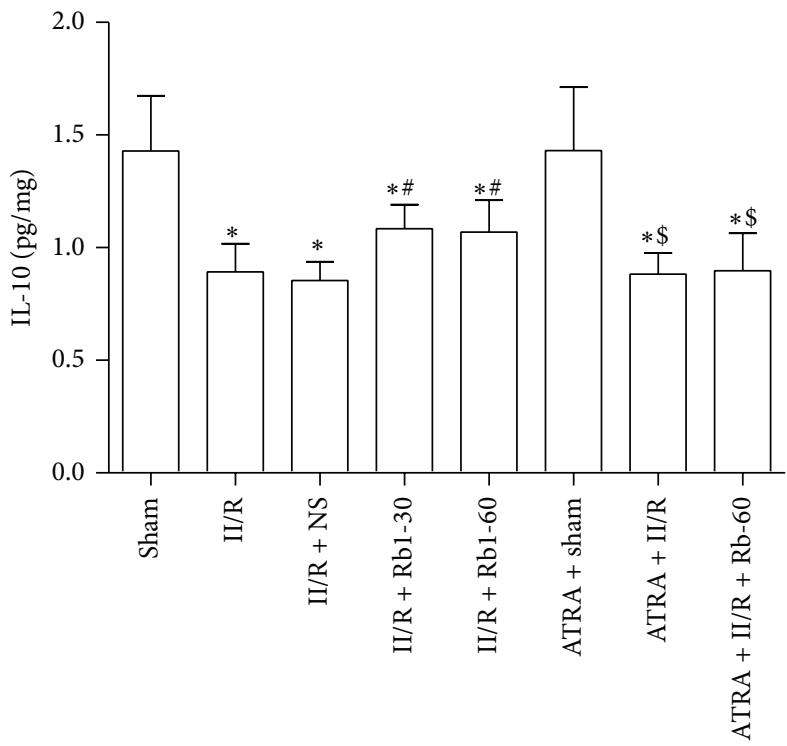

(c)

FIGURE 6: Cytokine levels in lung from mice. Cytokine levels were determined in lung homogenate using multiplex analysis: (a) TNF- $\alpha$, (b) IL-6, and (c) IL-10. Data are mean \pm SD, $n=10 ;{ }^{*} P<0.05$ versus Sham group, ${ }^{\#} P<0.05$ versus II/R group, and ${ }^{\$} P<0.05$ versus II/R + Rb1-60 group.

3.7. Effects of Ginsenoside Rb1 on Cytoplasmic HO-1 and Nuclear Nrf2 Expression in Lung Tissue by Western Blotting Analysis. To further confirm the protective effect of ginsenoside $\mathrm{Rb} 1$ on the lung tissue against II/R injury, protein expression of nuclear Nrf2 and cytoplasmic HO-1 was examined by Western blot. As shown in Figure 9, Nrf2 and HO-1 expression were both increased markedly in the II/R group as compared with the Sham group. II/R with Rb1 intervention further increased the expression of Nrf2 and HO-1 significantly. ATRA administration has no effects on the cytoplasmic HO-1 expression as compared with the Sham group. This indicated that Rb1 induced cytoplasmic HO-1 expression was inhibited by ATRA. There was no significant difference in Nrf2 expression between the ATRA + II/R group and the II/R group or between the ATRA + II/R + Rb1-60 group and the II/R group.

\section{Discussion}

In this study, we have demonstrated in a mice model that $45 \mathrm{~min}$ occlusion of SMA followed by $2 \mathrm{~h}$ of reperfusion caused significant lung injury as evidenced by pathologic morphologic changes seen in the lung tissue, as well as the increased lung wet/dry ratio, which is in accordance with the previous reports [25]. We found that postischemia treatment 


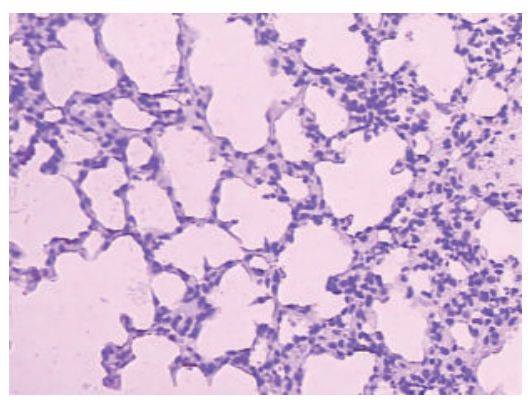

(a)

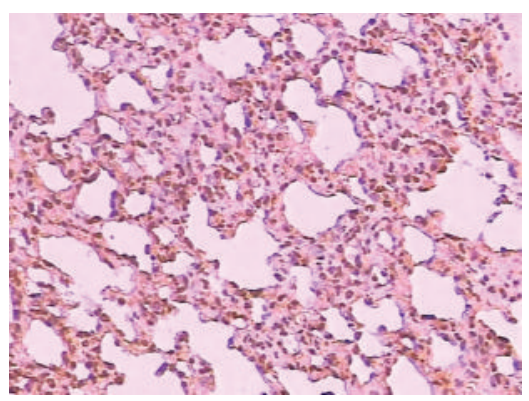

(d)

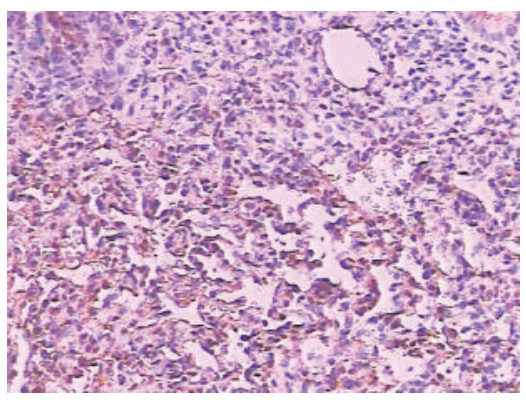

(b)

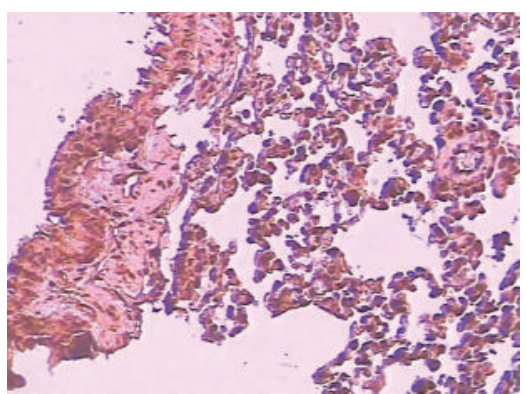

(e)

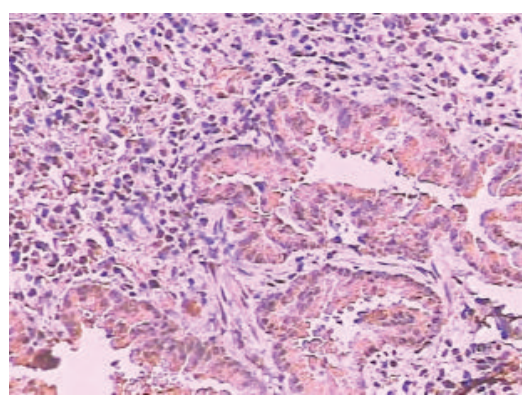

(c)

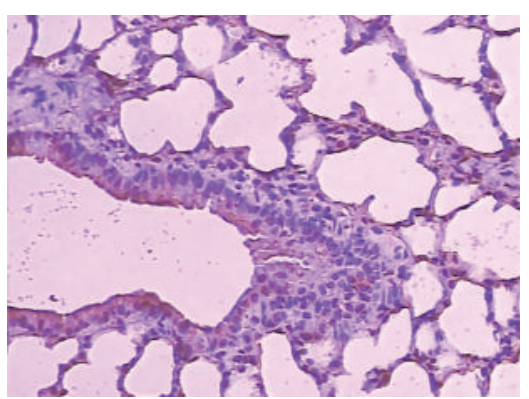

(f)

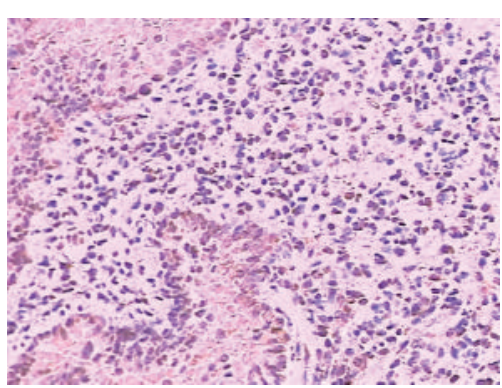

(g)

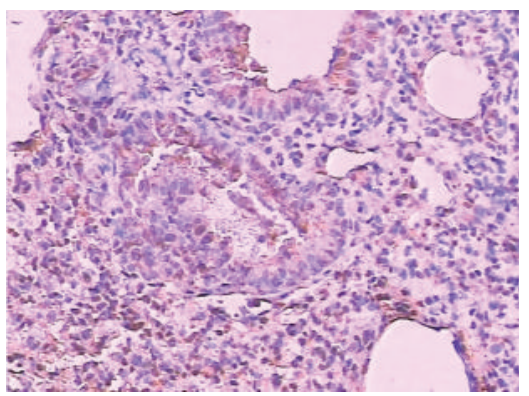

(h)

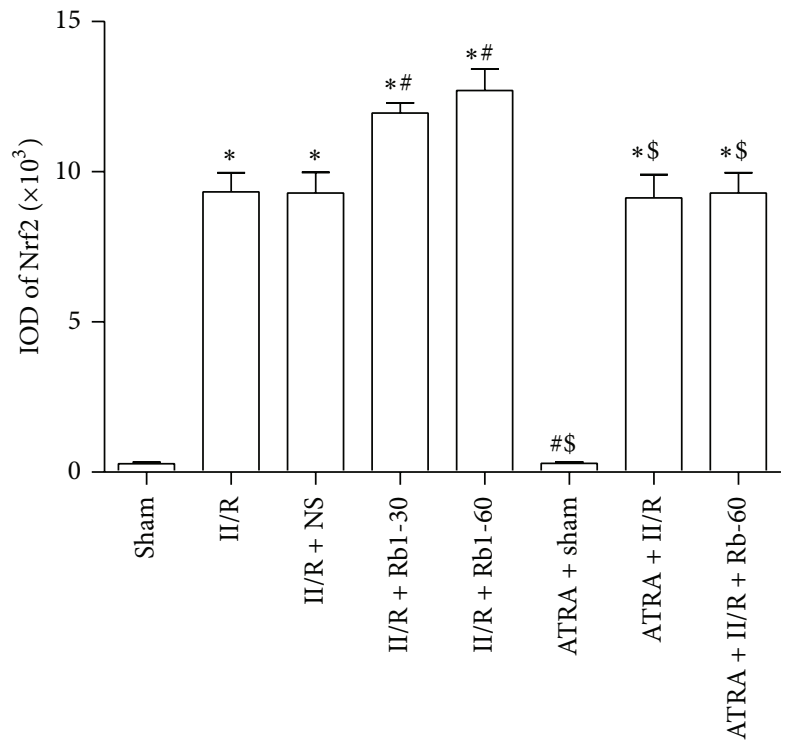

(i)

Figure 7: Expression of Nrf2 in the lung tissue under the light microscope (streptavidin-biotin complex immunohistochemistry, $\times 200)$. (a) Sham group, (b) II/R group, (c) II/R + NS group, (d) II/R + Rb1-30 group, (e) II/R + Rb1-60 group, (f) ATRA + Sham group, (g) ATRA + II/R group, and (h) ATRA + II/R + Rbl-60 group. Data are mean $\pm \mathrm{SD}, n=5 ;{ }^{*} P<0.05$ versus Sham group, ${ }^{\#} P<0.05$ versus II/R group, and ${ }^{\$} P<0.05$ versus II/R + Rb1-60 group. 


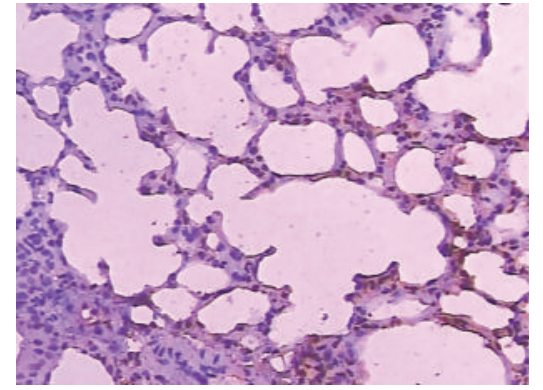

(a)

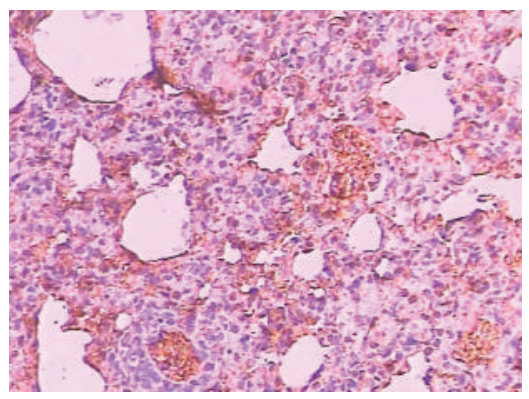

(d)

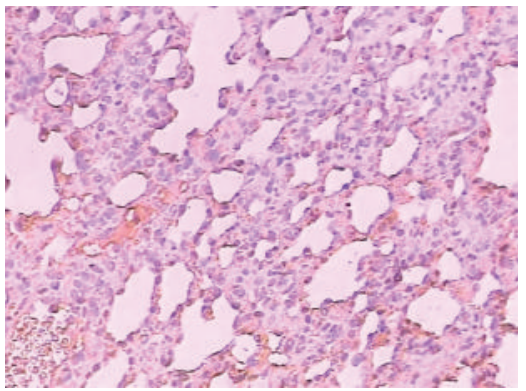

(b)

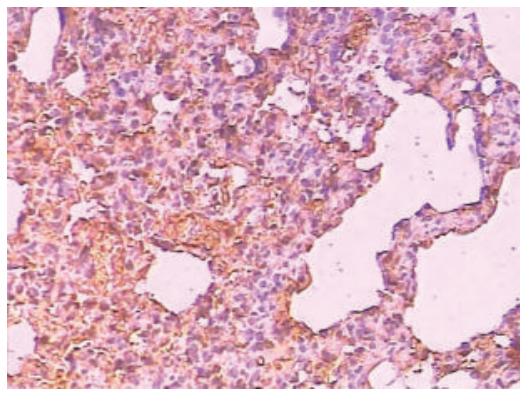

(e)

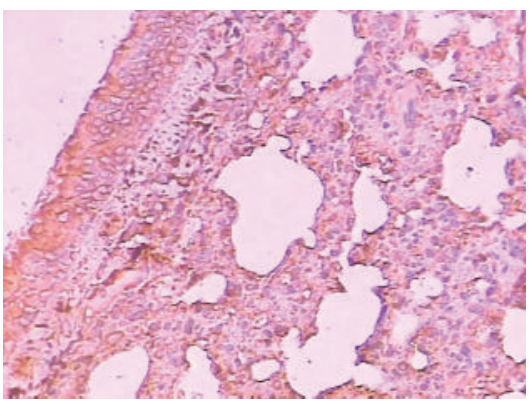

(c)

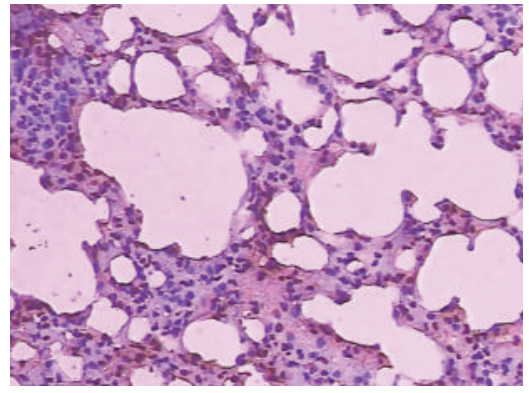

(f)

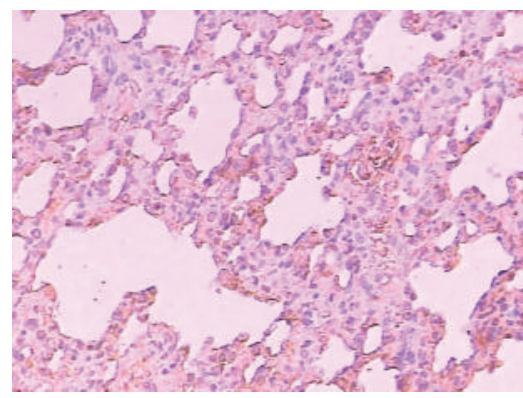

(g)

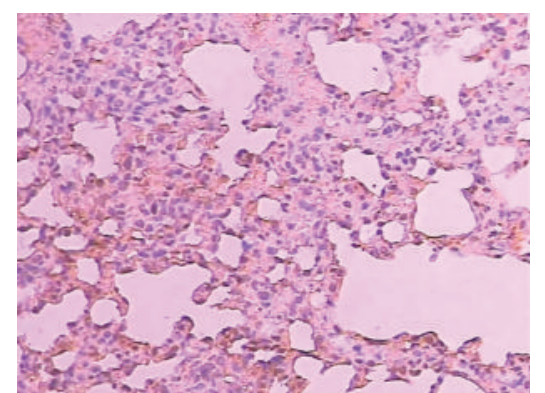

(h)

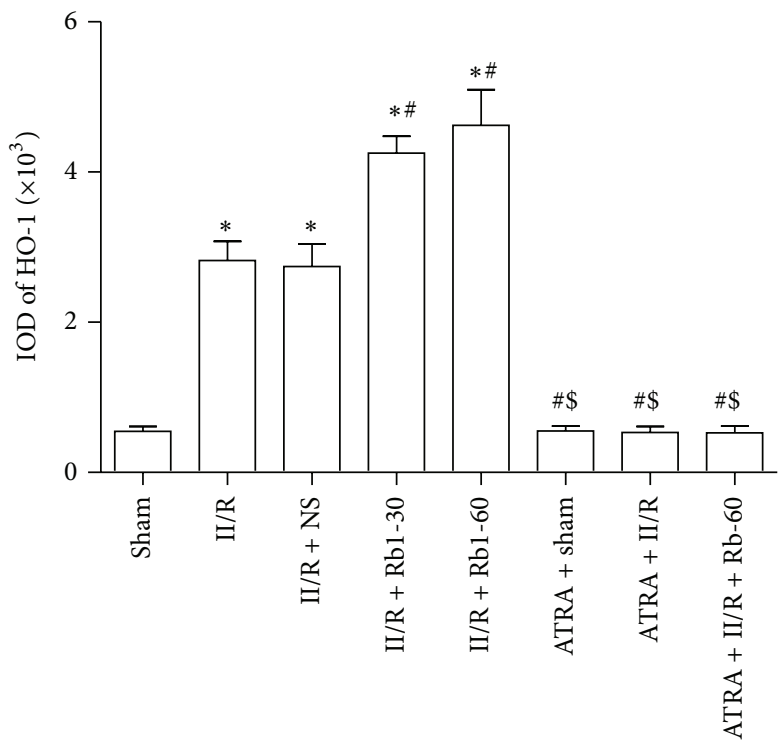

(i)

FIGURE 8: Expression of HO-1 in the lung tissue under the light microscope (streptavidin-biotin complex immunohistochemistry, $\times 200)$. (a) Sham group, (b) II/R group, (c) II/R + NS group, (d) II/R + Rb1-30 group, (e) II/R + Rb1-60 group, (F) ATRA + Sham group, (g) ATRA + II/R group, and (h) ATRA + II/R + Rbl-60 group. Data are mean $\pm \mathrm{SD}, n=5 ;{ }^{*} P<0.05$ versus Sham group, ${ }^{\#} P<0.05$ versus II/R group, and ${ }^{\$} P<0.05$ versus II/R + Rb1-60 group. 
Nrf2

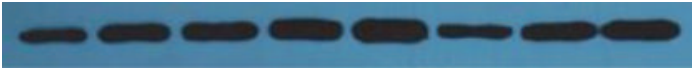

Lamin B1

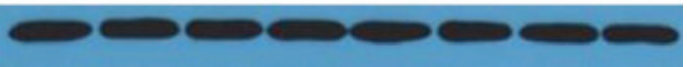

(a)

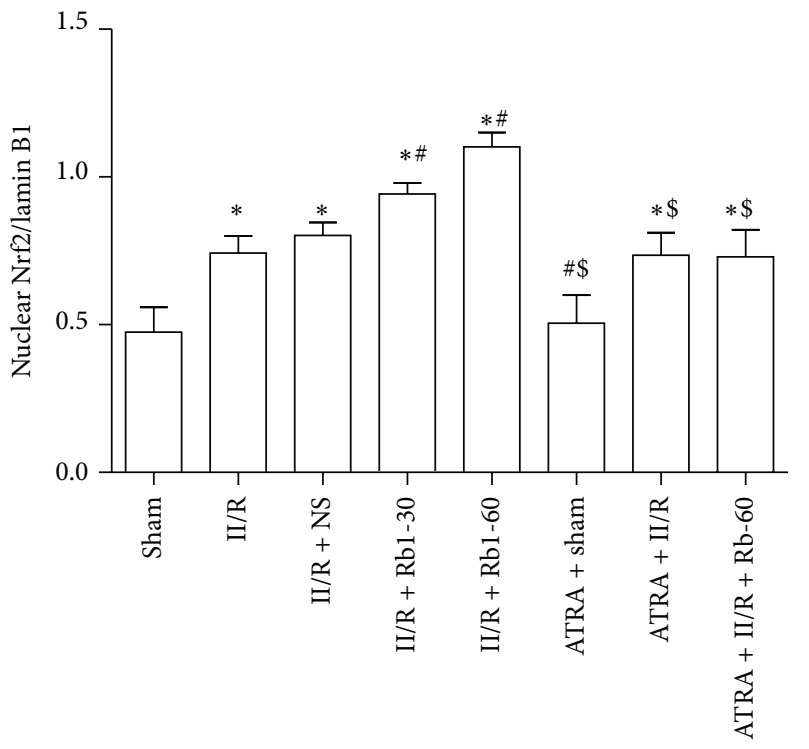

(c)
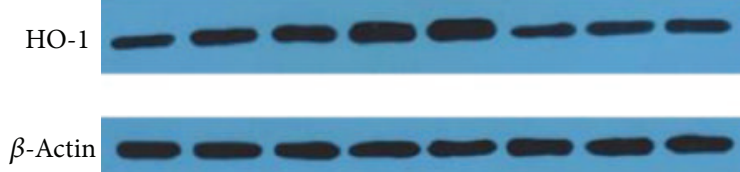

(b)

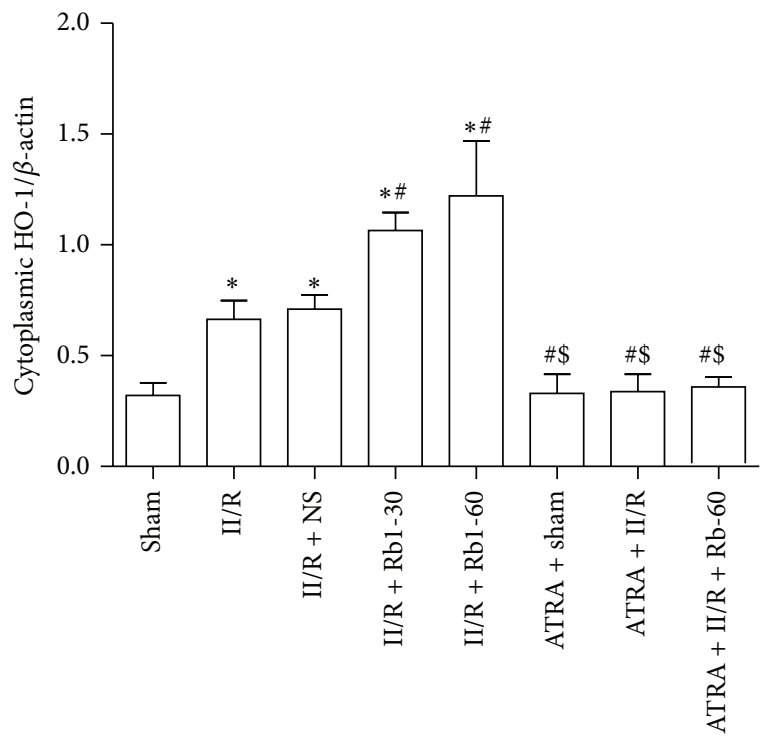

(d)

FIGURE 9: Western blotting analysis of the presence of Nrf2 in nuclear proteins and HO-1 in cytoplasmic proteins in the mice lung tissue. Data obtained from quantitative densitometry were presented as mean \pm SD. $n=10,{ }^{*} P<0.05$ versus Sham group, ${ }^{\#} P<0.05$ versus II/R group, and ${ }^{\$} P<0.05$ versus II/R + Rb1-60 group.

with ginsenoside Rb1 enhanced Nrf2 translocation to the nucleus in the lung tissues of mice and Rbl treatment could reduce pulmonary morphologic damage, alleviate injuries induced by oxidative stress, and modulate inflammatory reactions. Further, Nrf2 function inhibition with ATRA reverted the pulmonary protective effects of ginsenoside $\mathrm{Rb}$, indicating that ginsenoside Rb1 confers its respiratory protection via Nrf2/ARE signaling in the II/R induced acute lung injury.

The mechanisms of acute lung injury induced by II/R are complex. It is thought that the damage of intestinal mucosal barrier following II/R causes the dislocation of bacteria or endogenous endotoxin, thus leading to increased oxidative stress and systemic inflammatory reaction, which is one of the main reasons for acute lung injury.

Ginseng is one of the most widely used herbal medicines. Ginsenosides, the major active ingredient of ginseng, have been noticed for their multiple pharmacological effects on antioxidation, signal transduction pathways, and interaction with receptors [26]. Oxidative stress refers to the mismatched redox equilibrium between the production of free radicals and the ability of cells to defend against them. One feasible way to prevent free radical mediated cellular injuries is to augment the oxidative defense capacity through intake of antioxidants. Moreover, the induction of endogenous phase II detoxifying enzymes or antioxidative proteins seems to be a reasonable strategy for delaying disease progression.
Activation of Nrf2/ARE plays an important role in protecting cells from oxidative stress $[27,28]$. The ability of Nrf2 to upregulate the expression of antioxidant genes via ARE suggests that increasing Nrf2 activity may provide a useful system for combating oxidative insults. Several recent reports have demonstrated that coordinate upregulation of AREdriven genes protects organs from ischemia reperfusion injury [29-31]. Accumulating evidence also suggests that upregulation of HO-1 expression and the subsequent increase in $\mathrm{HO}$ activity may confer an adaptive survival response against oxidative insults. Our previous studies showed that $\mathrm{Rb} 1$ reduces renal apoptosis and alleviates renal dysfunction after II/R in part through the Nrf2/ARE pathway [32]. Wang et al. demonstrated that $\mathrm{Rbl}$ attenuates lung injury induced by II/R via inhibiting NF- $\kappa \mathrm{B}$ activation [33]. To determine the mechanism by which postischemia treatment with $\mathrm{Rb} 1$ reduces II/R-induced ROS generation, we examined the effect of ginsenoside $\mathrm{Rb} 1$ on Nrf2 and HO-1 expression in mice lung tissues. Our present study demonstrated that Rb1 increased nuclear $\mathrm{Nrf} 2$ protein and cytoplasmic $\mathrm{HO}-1$ protein expressions in lung tissues of mice after II/R. The increase of HO-1 expression by Rb1 conferred cytoprotection against II/R induced oxidative stress. In addition, previous studies have shown that ATRA does not affect the half-life of Nrf2 or its nuclear translocation. ATRA inhibits Nrf2 function by stimulating the formation of $\operatorname{Nrf} 2$ :RAR $\alpha$-containing complexes that do not bind to the ARE [18]. We showed that ATRA, as a 
potent inhibitor for combination of Nrf2 with ARE, partially reversed the protective effects of $\mathrm{Rbl}$, thus providing further evidence for Nrf2/ARE as a possible cytoprotective pathway for Rbl.

Ginseng extract was reported to have immunomodulatory effects [34]. Smolinski and Pestka [35] reported that immunologic effects include modulation of lipopolysaccharide-induced proinflammatory cytokine production in vitro and in vivo by ginsenoside Rb1. This was also confirmed with our study in which Rbl significantly reduced the tissue level of TNF- $\alpha$, IL- 6 , and IL-10. These results show that Rb1 may have multiple mechanisms of action that affect cytoprotection by both reducing ROS generation and increasing the anti-inflammatory effect.

In summary, our present study indicates that ginsenoside $\mathrm{Rb} 1$ alleviates acute lung injury following II/R via activating Nrf2/ARE pathway. The experiment data may help us further understand the pharmacological effects of Rb1 and also suggest a new therapeutic target to protect the body from II/R injury. However, further studies need to be performed in transfection of lung endothelial cells and intestinal epithelial cells with the siRNA and expressing plasmid for Nrf2 to confirm the findings of the current study.

\section{Disclosure}

All authors have no financial, personal, or other relationships with other people or organizations that could inappropriately influence the work.

\section{Conflict of Interests}

The authors declare that they have no conflict of interests.

\section{Authors' Contribution}

Ying Jiang and Zhen Zhou contributed equally to this work.

\section{Acknowledgment}

This study was mainly supported by a grant from the National Natural Science Foundation of China (NSFC) 81170768 and partly by NSFC 81471844 and 81300674 .

\section{References}

[1] H. T. Hassoun, B. C. Kone, D. W. Mercer, F. G. Moody, N. W. Weisbrodt, and F. A. Moore, "Post-injury multiple organ failure: the role of the gut," Shock, vol. 15, no. 1, pp. 1-10, 2001.

[2] A. Pierro and S. Eaton, "Intestinal ischemia reperfusion injury and multisystem organ failure," Seminars in Pediatric Surgery, vol. 13, no. 1, pp. 11-17, 2004.

[3] T.-H. Lan, Z.-W. Xu, Z. Wang, Y.-L. Wu, W.-K. Wu, and H.-M. Tan, "Ginsenoside Rbl prevents homocysteine-induced endothelial dysfunction via PI3K/Akt activation and PKC inhibition," Biochemical Pharmacology, vol. 82, no. 2, pp. 148155, 2011.

[4] Y. P. Hwang and H. G. Jeong, "Ginsenoside Rb1 protects against 6-hydroxydopamine-induced oxidative stress by increasing heme oxygenase-1 expression through an estrogen receptor-related PI3K/Akt/Nrf2-dependent pathway in human dopaminergic cells," Toxicology and Applied Pharmacology, vol. 242, no. 1, pp. 18-28, 2010.

[5] Y. Wu, Z.-Y. Xia, J. Dou et al., "Protective effect of ginsenoside Rbl against myocardial ischemia/reperfusion injury in streptozotocin-induced diabetic rats," Molecular Biology Reports, vol. 38, no. 7, pp. 4327-4335, 2011.

[6] Z. Jiang, Y. Wang, X. Zhang et al., "Preventive and therapeutic effects of ginsenoside Rb1 for neural injury during cerebral infarction in rats," The American Journal of Chinese Medicine, vol. 41, no. 2, pp. 341-352, 2013.

[7] L. Shen, Y. Xiong, D. Q.-H. Wang et al., "Ginsenoside Rb1 reduces fatty liver by activating AMP-activated protein kinase in obese rats," Journal of Lipid Research, vol. 54, no. 5, pp. 14301438, 2013.

[8] R. Hashimoto, J. Yu, H. Koizumi, Y. Ouchi, and T. Okabe, "Ginsenoside Rb1 prevents $\mathrm{MPP}^{+}$-induced apoptosis in PC12 cells by stimulating estrogen receptors with consequent activation of ERK1/2, Akt and inhibition of SAPK/JNK, p38 MAPK," Evidence-Based Complementary and Alternative Medicine, vol. 2012, Article ID 693717, 8 pages, 2012.

[9] D. Liu, H. Zhang, W. Gu, Y. Liu, and M. Zhang, "Neuroprotective effects of ginsenoside rbl on high glucose-induced neurotoxicity in primary cultured rat," PLoS ONE, vol. 8, no. 11, Article ID e79399, 2013.

[10] P. Yao, A. Nussler, L. Liu et al., "Quercetin protects human hepatocytes from ethanol-derived oxidative stress by inducing heme oxygenase-1 via the MAPK/Nrf2 pathways," Journal of Hepatology, vol. 47, no. 2, pp. 253-261, 2007.

[11] A. S. Pachori, L. G. Melo, L. Zhang, S. D. Solomon, and V. J. Dzau, "Chronic recurrent myocardial ischemic injury is significantly attenuated by pre-emptive adeno-associated virus heme oxygenase-1 gene delivery," Journal of the American College of Cardiology, vol. 47, no. 3, pp. 635-643, 2006.

[12] S. Lee and K. Suk, "Heme oxygenase-1 mediates cytoprotective effects of immunostimulation in microglia," Biochemical Pharmacology, vol. 74, no. 5, pp. 723-729, 2007.

[13] H. J. Kang, Y. B. Hong, H. J. Kim, and I. Bae, "CR6-interacting factor 1 (CRIF1) regulates NF-E2-related factor 2 (NRF2) protein stability by proteasome-mediated degradation," Journal of Biological Chemistry, vol. 285, no. 28, pp. 21258-21268, 2010.

[14] J. W. Kaspar, S. K. Niture, and A. K. Jaiswal, "Nrf2:INrf2 (Keap1) signaling in oxidative stress," Free Radical Biology and Medicine, vol. 47, no. 9, pp. 1304-1309, 2009.

[15] S. Dhakshinamoorthy, D. J. Long II, and A. K. Jaiswal, "Antioxidant regulation of genes encoding enzymes that detoxify xenobiotics and carcinogens," Current Topics in Cellular Regulation, vol. 36, pp. 201-216, 2001.

[16] D. Bloom, S. Dhakshinamoorthy, W. Wang, C. M. Celli, and A. K. Jaiswal, "Role of NFE2 related factors in oxidative stress," in Cell and Molecular Responses to Stress. Volume 2: Protein Adaptation and Signal Transduction, K. B. Storey and J. M. Storey, Eds., pp. 229-238, Elsevier, Amsterdam, The Netherlands, 2001.

[17] Y.-F. Liao, W. Zhu, D.-P. Li, and X. Zhu, "Heme oxygenase-1 and gut ischemia/reperfusion injury: a short review," World Journal of Gastroenterology, vol. 19, no. 23, pp. 3555-3561, 2013.

[18] X. J. Wang, J. D. Hayes, C. J. Henderson, and C. R. Wolf, "Identification of retinoic acid as an inhibitor of transcription factor Nrf2 through activation of retinoic acid receptor alpha," Proceedings of the National Academy of Sciences of the United States of America, vol. 104, no. 49, pp. 19589-19594, 2007. 
[19] Q. Sun, Q. T. Meng, Y. Jiang, and Z.-Y. Xia, “Ginsenoside Rb1 attenuates intestinal ischemia reperfusion induced renal injury by activating Nrf2/ARE pathway," Molecules, vol. 17, no. 6, pp. 7195-7205, 2012.

[20] J. Shen, G. Fu, L. Jiang, J. Xu, L. Li, and G. Fu, "Effect of dexmedetomidine pretreatment on lung injury following intestinal ischemia-reperfusion," Experimental and Therapeutic Medicine, vol. 6, no. 6, pp. 1359-1364, 2013.

[21] C. J. Chiu, A. H. McArdle, R. Brown, H. J. Scott, and F. N. Gurd, "Intestinal mucosal lesion in low-flow states. I. A morphological, hemodynamic, and metabolic reappraisal," Archives of Surgery, vol. 101, no. 4, pp. 478-483, 1970.

[22] M. L. Pearce, J. Yamashita, and J. Beazell, "Measurement of pulmonary edema," Circulation Research, vol. 16, pp. 482-488, 1965.

[23] A. J. L. Chia, C. E. Goldring, N. R. Kitteringham, S. Q. Wong, P. Morgan, and B. K. Park, "Differential effect of covalent protein modification and glutathione depletion on the transcriptional response of Nrf2 and NF- $\kappa \mathrm{B}$," Biochemical Pharmacology, vol. 80, no. 3, pp. 410-421, 2010.

[24] K. X. Liu, Y. S. Li, W. Q. Huang, C. Li, and J.-X. Liu, "Immediate but not delayed postconditioning during reperfusion attenuates acute lung injury induced by intestinal ischemia/reperfusion in rats: comparison with ischemic preconditioning," Journal of Surgical Research, vol. 157, no. 1, pp. e55-e62, 2009.

[25] B. C. Guido, M. Zanatelli, W. Tavares-de-Lima, S. M. Oliani, and A. S. Damazo, "Annexin-A1 peptide down-regulates the leukocyte recruitment and up-regulates interleukin-10 release into lung after intestinal ischemia-reperfusion in mice," Journal of Inflammation, vol. 10, no. 1, article 10, 2013.

[26] J. M. Lü, Q. Yao, and C. Chen, "Ginseng compounds: an update on their molecular mechanisms and medical applications," Current Vascular Pharmacology, vol. 7, no. 3, pp. 293-302, 2009.

[27] W. F. Yao, G. J. Luo, G. S. Zhu et al., "Propofol activation of the Nrf2 pathway is associated with amelioration of acute lung injury in a rat liver transplantation model," Oxidative Medicine and Cellular Longevity, vol. 2014, Article ID 258567, 9 pages, 2014.

[28] H. R. Potteti, N. M. Reddy, T. K. Hei, D. V. Kalvakolanu, and S. P. Reddy, "The NRF2 activation and antioxidative response are not impaired overall during hyperoxia-induced lung epithelial cell death," Oxidative Medicine and Cellular Longevity, vol. 2013, Article ID 798401, 11 pages, 2013.

[29] B. Ke, X.-D. Shen, Y. Zhang et al., "KEAP1-NRF2 complex in ischemia-induced hepatocellular damage of mouse liver transplants," Journal of Hepatology, vol. 59, no. 6, pp. 1200-1207, 2013.

[30] W. Wang, J. Kang, H. Li et al., "Regulation of endoplasmic reticulum stress in rat cortex by p62/ZIP through the Keap1Nrf2-ARE signalling pathway after transient focal cerebral ischaemia," Brain Injury, vol. 27, no. 7-8, pp. 924-933, 2013.

[31] B. F. Peake, C. K. Nicholson, J. P. Lambert et al., "Hydrogen sulfide preconditions the $\mathrm{db} / \mathrm{db}$ diabetic mouse heart against ischemia-reperfusion injury by activating Nrf2 signaling in an Erk-dependent manner," The American Journal of PhysiologyHeart and Circulatory Physiology, vol. 304, no. 9, pp. H1215H1224, 2013.

[32] Q. Sun, Q. T. Meng, Y. Jiang et al., "Protective effect of ginsenoside Rbl against intestinal ischemia-reperfusion induced acute renal injury in mice," PLoS ONE, vol. 8, no. 12, Article ID e80859, 2013.
[33] J. Wang, L. Qiao, S. Li, and G. Yang, "Protective effect of ginsenoside Rbl against lung injury induced by intestinal ischemiareperfusion in rats," Molecules, vol. 18, no. 1, pp. 1214-1226, 2013.

[34] A. Rhule, B. Rase, J. R. Smith, and D. M. Shepherd, “Toll-like receptor ligand-induced activation of murine DC2.4 cells is attenuated by Panax notoginseng," Journal of Ethnopharmacology, vol. 116, no. 1, pp. 179-186, 2008.

[35] A. T. Smolinski and J. J. Pestka, "Modulation of lipopolysaccharide-induced proinflammatory cytokine production in vitro and in vivo by the herbal constituents apigenin (chamomile), ginsenoside $\mathrm{Rb}_{1}$ (ginseng) and parthenolide (feverfew)," Food and Chemical Toxicology, vol. 41, no. 10, pp. 1381-1390, 2003. 


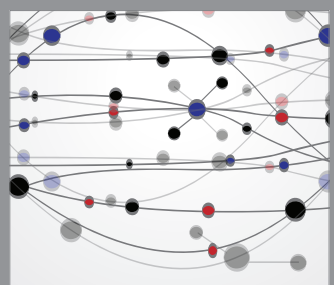

The Scientific World Journal
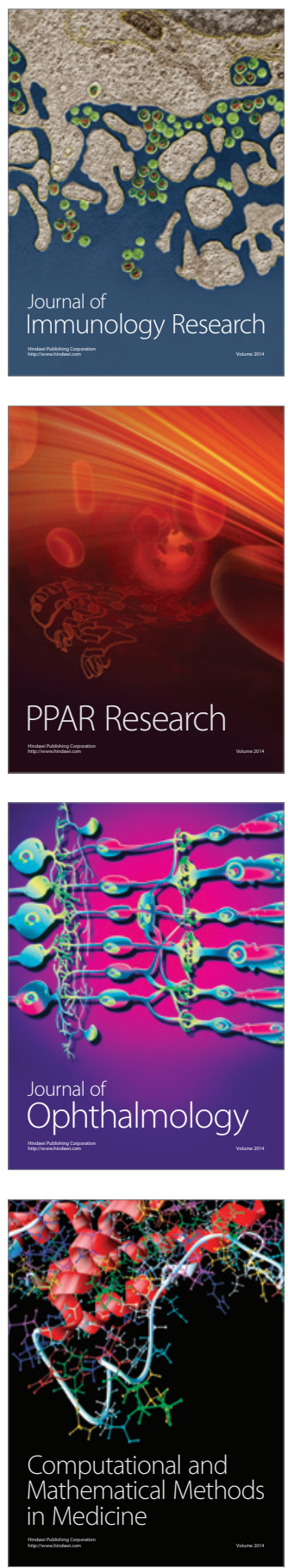

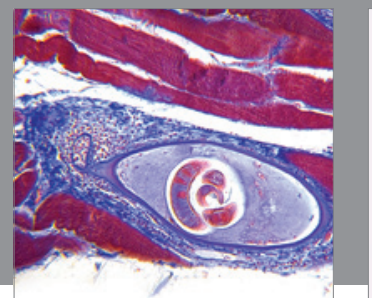

Gastroenterology

Research and Practice
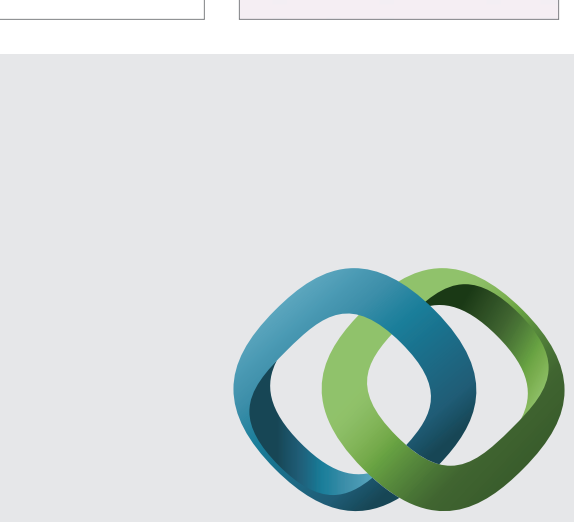

\section{Hindawi}

Submit your manuscripts at

http://www.hindawi.com
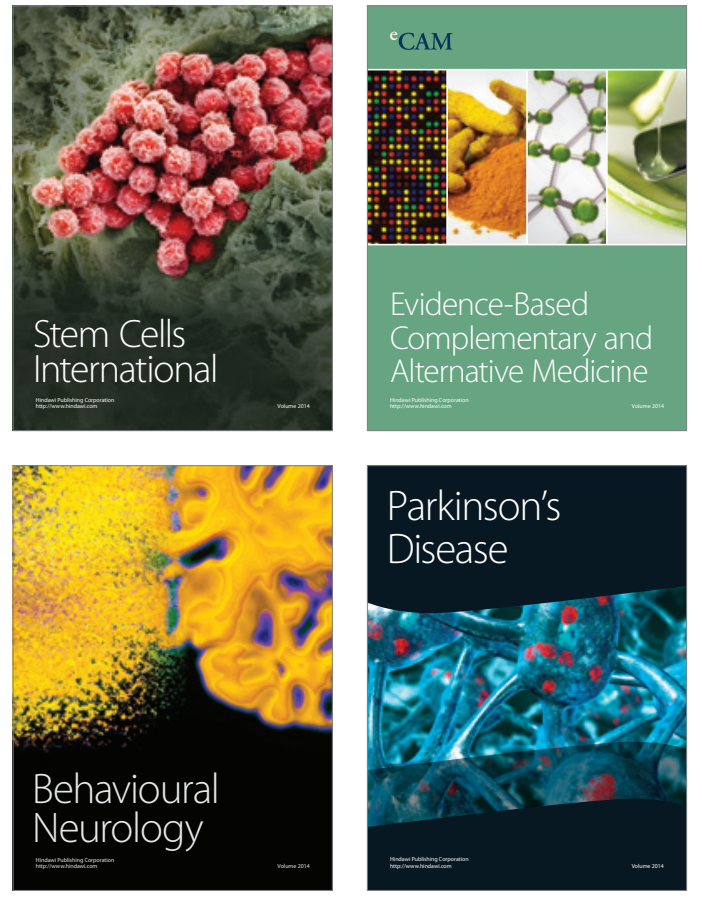
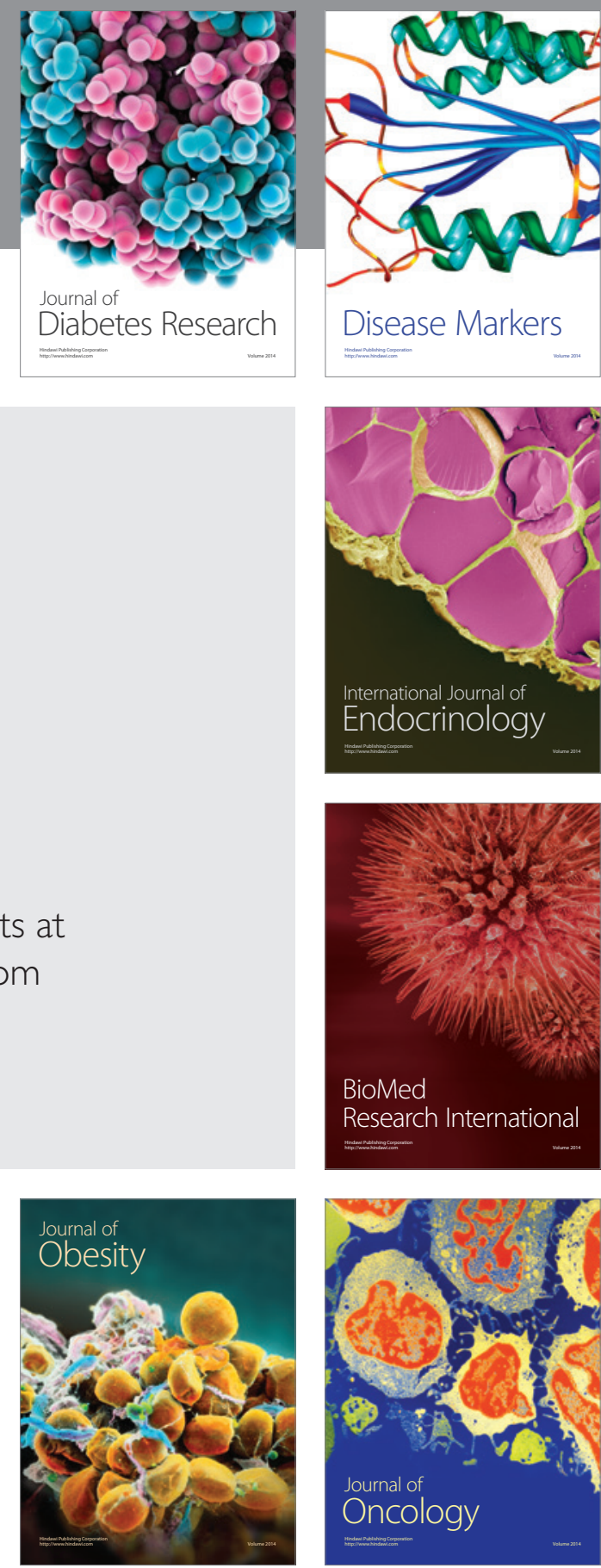

Disease Markers
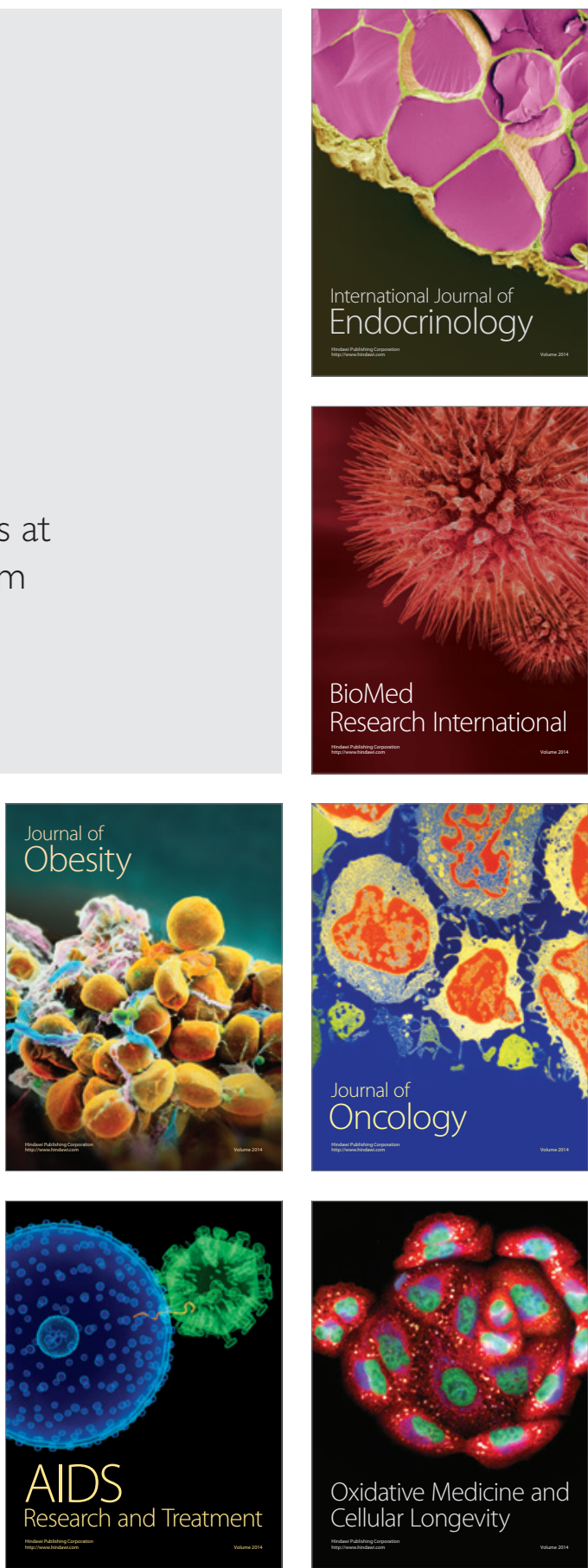